\title{
The Comparison for Serratus Anterior Muscle Activity during Protraction in Open Chain and Closed Chain Exercises in Healthy Adults
}

\author{
Tae-ho Kim, Ph.D.; Han-kyu Park, Ph.D. \\ Department of Physical Therapy, College of Rehabilitation Science, Daegu University, Daegu, South Korea
}

Background In shoulder dysfunction, shoulder pain, and glenohumeral joint impingement, it is necessary to activate the serratus anterior muscle.

Purpose This study aimed to compare serratus anterior muscle activity during protraction in open chain and close chain exercises in order to determine the posture that is best at selectively activating the serratus anterior muscle.

Study design One group, two condition design.

Methods Thirteen healthy university students participated in this study. The muscle activities of the serratus anterior, the upper trapezius, and the pectoralis major during protraction of scapula were recorded using a wireless surface electromyography system. The subjects performed protraction of scapula on both open chain exercise in supine posture and closed chain exercise in quadruped posture. To evaluate the selective activation of the serratus anterior muscle, the ratio of the serratus anterior activation versus the upper trapezius activation and the ratio of the serratus anterior activation versus the pectoralis major activation were calculated.

Results The muscle activity of serratus anterior was significantly higher and pectoralis major was significantly lower the open chain exercise rather than the closed chain exercise. The muscle activity of upper trapezius was not significantly difference between two exercises. Also, each ratio of serratus anterior against other muscles was significantly high in open chain exercise.

Conclusions This study's findings suggest that the open chain exercise in supine posture is better than the closed chain exercise in quadruped posture for selective activation of the serratus anterior muscle.

Key words Closed chain exercise; Open chain exercise; Push-up; Serratus anterior; Winging scapula.

\section{INTRODUCTION}

Alignment is an indicator of possible changes in muscle length and the joint alignments in the shoulder joint. Abnormal scapular alignment is a common cause of the shoulder pain. ${ }^{1}$ The impairments of the alignment in the scapula impede optimal movement in the shoulder joint. ${ }^{2}$

The serratus anterior muscle is the most important muscle to maintain good scapular alignment in the shoulder joint.
Journal of KEMA 2018; 2(1): $1-5$ Published Online June 30, 2018 pISSN: $2586-4351$ eISSN: 2586-5706

Article History Received 21 April 2018 Revised 01 May 2018 Accepted 11 May 2018

\section{CONTACT}

hanqy@naver.com Han-kyu Park, Department of Physical Therapy, College of Rehabilitation Science, Daegu University, Daegu, South Korea

\section{This is an Open-Access article under the terms of the Creative Commons Att- ribution Non-Commercial Li- cense (http://creativecommons org/licenses/by-nc/4.0) which permits unrestricted non-co- mmercial use, distribution, and reproduction in any medium, provided the original work is properly cited.}

The serratus anterior is an important scapular stabilizer during flexion or abduction of shoulder joint; it helps prevent anterior tilting or winging of the scapula. ${ }^{3}$ Moreover, the serratus anterior can contribute to all components of normal three-dimensional movement of the scapula on the thorax when elevating the arm. ${ }^{4}$

In shoulder dysfunction, shoulder pain, and glenohumeral joint impingement, it is necessary to activate the serratus anterior muscle. Many previous studies have investigated 
various exercises to determine the most effective method to activate the serratus anterior muscle. ${ }^{5-9}$ Decker et al. reported that Push-up, punch exercises, and dynamic hug activate the serratus anterior muscle. ${ }^{6}$ Another study reported that performing push-up exercises on a stable surface can produce higher levels of serratus anterior activity and lower levels of trapezius muscle activity in comparison to unstable surfaces. ${ }^{10}$ A different study found that standard push-up plus is the most optimal exercise for maximum activation of the serratus anterior with minimal activation of the upper trapezius in comparison to knee push-up plus, elbow pushup plus, and wall push-up plus exercises. ${ }^{8}$ Many studies have investigated activating the serratus anterior muscle during closed chain exercises, which are a type of push-up exercise. Closed chain exercises involve motions in which the body moves on a distal segment that is fixed or stabilized on a support surface; thus, closed chain exercises are typically performed in weight-bearing positions using quadruped press-ups from a chair, wall push-offs, or prone pushups. ${ }^{11}$ The selection in open chain or closed chain exercises is based on the goals of an individualized therapeutic program and an analysis of the benefits and limitations in two forms of exercise. ${ }^{11}$

This study aimed to compare serratus anterior muscle activity during protraction in open chain and close chain exercises in order to determine the posture that is best at selectively activating the serratus anterior muscle.

\section{METHODS}

\section{Participants}

Thirteen healthy university students participated in this study ( 9 males and 4 females). The subjects were provided a written informed consent according to the ethical standards of the Declaration of Helsinki, and they agreed to participate in this study voluntarily. The characteristics of subjects were mean age of $22.92 \pm 2.14$ years, mean height of 172.06 $\pm 9.16 \mathrm{~cm}$, and a mean weight of $67.23 \pm 11.66 \mathrm{~kg}$. And distance from spine to median board of scapula (winging scapula) were $8.67 \pm 0.86$. The exclusion criteria were past or present musculoskeletal conditions affecting the glenohumeral joint, rotator cuff muscles, or acromioclavicular joints, as well as neurological disorders.

\section{Measurement of muscle activity}

The muscle activities during protraction of scapular were recorded using a wireless surface electromyography system (TeleMyo DTS, Noraxon, Scottsdale, Arizona, USA) with a sampling frequency of $1000 \mathrm{~Hz}$. A bandpass filter of surface electromyography was used between 20 and $400 \mathrm{~Hz}$ and a
$60 \mathrm{~Hz}$ for notch filters. Before placing EMG electrodes, all sites for the attaching point were shaved with razors and deterged with an alcohol-soaked paper towel. The electrodes for serratus anterior were attached to the muscle belly on the midaxillary line of the right fifth rib, the upper trapezius were attached to muscle belly at the midpoint between the $\mathrm{C} 7$ spinous process and the right acromioclavicular joint, which is the region of insertion of the trapezius and the sternal fiber of pectoralis major were attached to chest wall horizontal from the arising muscle mass approximately $2 \mathrm{~cm}$ out from the axillary fold. ${ }^{12}$

Before the measurements were obtained, the maximal voluntary isometric contraction (MVIC) was measured for the serratus anterior, the upper trapezius, and the pectoralis major muscles to normalize electromyography (EMG) activity. The examiner asked each subject to perform a 5second MVIC three times for each muscle against manual resistance from the examiner. The measurement positions for MVIC were based on the manual muscle testing recommended by Kendall. ${ }^{13}$ The present study collected EMG data for the middle 3 seconds. The MVIC value used the average root mean square (RMS) of the three trials. The average EMG activity was expressed as a percentage of the MVIC value (\%MVIC). A 1-minute interval was allowed between each contraction to avoid muscle fatigue. To evaluate the selective activation of the serratus anterior muscle, the ratio of the serratus anterior activation versus the upper trapezius activation (serratus anterior/upper trapezius) and the ratio of the serratus anterior activation versus the pectoralis major activation (serratus anterior/pectoralis major) were calculated.

\section{Procedure}

The subjects performed protraction of the scapula in both an open chain posture and a closed chain posture. The position for the open chain exercise was shoulder flexion at a 90-degree angle with elbow extension in a supine position. Each subject pushed his/her arm toward the ceiling to protract the scapula and held this position for 5 seconds (Figure 1a). A quadruped position was used for the closed chain exercise. Each subject was on his hands and knees with his feet pointing away from body, and his hips centered over the knees, which were spaced a few inches apart. The hips were positioned at a 90-degree angle, the subject's spine was straight, the shoulders were centered over the hands, and the head was in-line with the body. The subject pushed his/her arm toward the floor to protract the scapula and then held this position for 5 seconds (Figure $1 b$ ). The order of the exercise was determined randomly by coin toss. The interval resting time between the exercise postures was set 


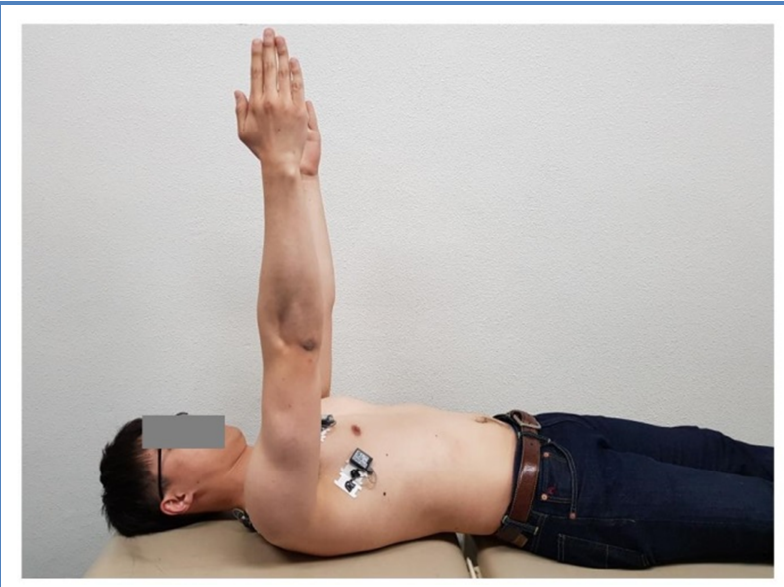

(a) Open chain exercise

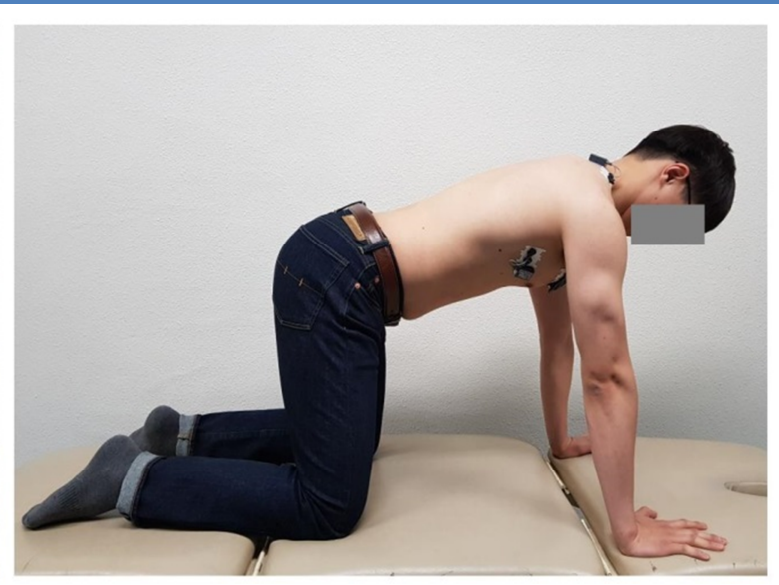

(b) Closed chain exercise

Figure 1. Measurement of muscle activities in open chain and closed chain exercise.

to 10 minutes in order to minimize the muscle fatigue of the subjects.

\section{Statistical analysis}

The data normality was tested using the Shapiro-Wilk test. The paired $t$-test was used to compare the differences in the muscle activities of the serratus anterior, upper trapezius, and pectoralis major, as well as the ratio of the serratus anterior activity versus the activity of the other two muscles during protraction when the subjects held the open chain and closed chain postures. The level of statistical significance was set at $p<0.05$. The Statistical Package for the Social Sciences (SPSS) version 23.0 (SPSS, Inc., Chicago, IL, USA) software was used for the statistical analysis.

\section{RESULTS}

The muscle activity of the serratus anterior was significantly higher in the open chain exercise than in the closedchain exercise, and the activity of the pectoralis major was significantly lower in the open chain exercise than the closed chain exercise $(p<0.05)$ (Table 1)(Figure 2a). The ratio of the serratus anterior activity versus the upper trapezius activity and the ratio of the serratus anterior activity versus the pectoralis major activity were significantly higher in the open chain exercise than the closed chain exercise $(p<0.05)$ (Table 1)(Figure 2b).

\section{DISCUSSION}

This study aimed to compare the muscle activities of the serratus anterior, upper trapezius, and pectoralis major during protraction in open chain and closed chain exercise postures with 13 healthy university students in order to determine which posture selectively activated the serratus anterior muscle. The study results showed that the muscle activity of the serratus anterior was significantly higher and the muscle activity of the pectoralis major was significantly lower in the open chain exercise in comparison to the closed chain exercise. No significant difference between the two exercises was found for the muscle activity of the upper trapezius. Moreover, in the open chain exercise, the ratio of serratus anterior activation versus activation of the other two muscles was significantly high. This study found that the open chain exercise in the supine posture selectively activates the serratus anterior better than the closed chain exercise in the quadruped posture.

In order to enhance activation of the serratus anterior, ei-

Table 1. Comparison for muscle activities on open chain and closed chain exercise (unit : \%MVIC, N=13)

\begin{tabular}{ccccc}
\hline Muscles & Open chain exercise & Closed chain exercise & $t$ & $p$ \\
\hline Serratus anterior & $42.08 \pm 20.44$ & $12.65 \pm 6.37$ & -6.00 & 0.00 \\
Upper trapezius & $5.13 \pm 2.45$ & $4.36 \pm 1.6$ & -1.00 & 0.33 \\
Pectoralis major & $6.94 \pm 3.23$ & $20.91 \pm 8.06$ & 7.17 & 0.00 \\
A ratio Serratus anterior/ & $10.47 \pm 8.54$ & $3.31 \pm 1.92$ & -3.03 & 0.01 \\
$\quad \begin{array}{c}\text { upper trapezius } \\
\text { A ratio Serratus anterior/ } \\
\text { pectoralis major }\end{array}$ & $7.62 \pm 5.86$ & $0.63 \pm 0.25$ & -4.38 & 0.00 \\
\hline
\end{tabular}




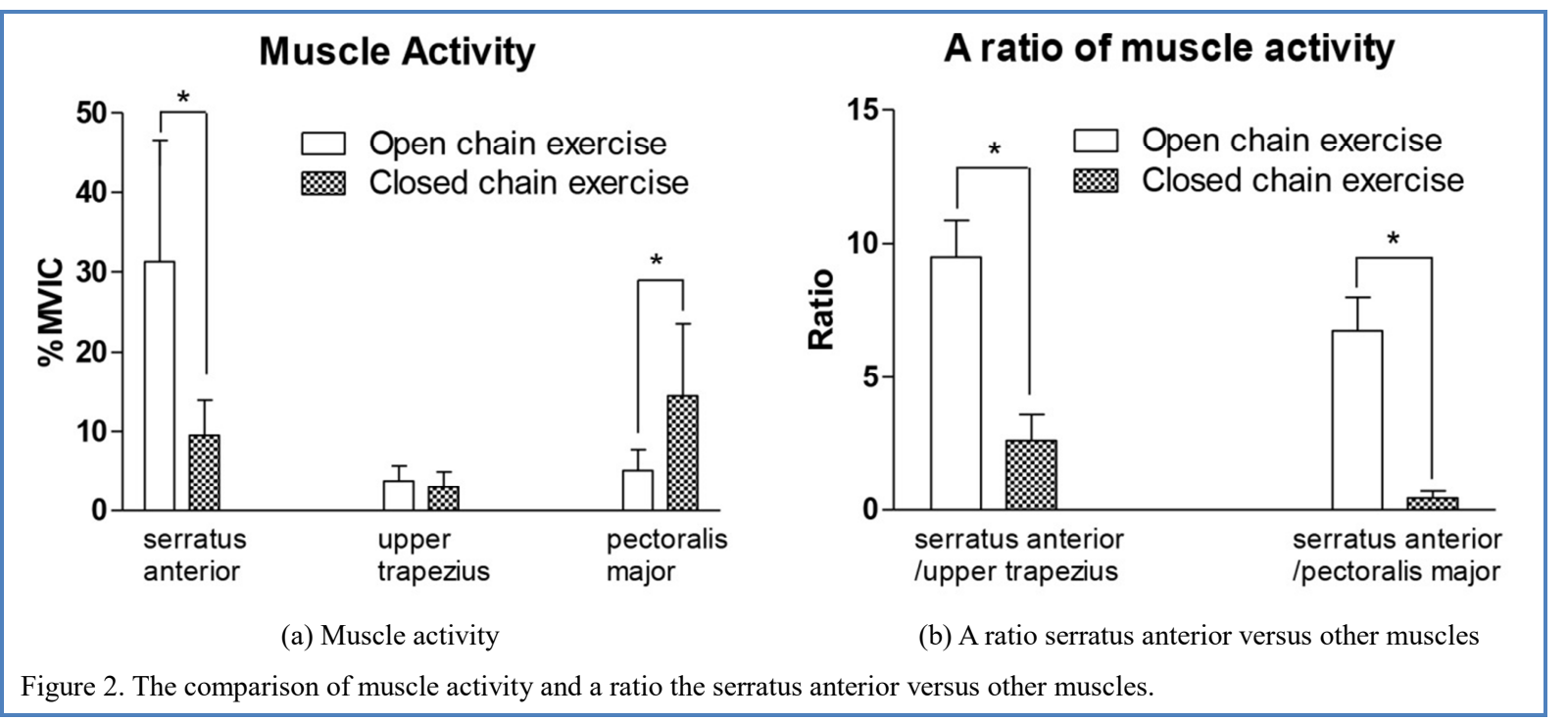

ther upward rotation or protraction of the scapula, which is possible with push-up exercises, was performed. These movements are also used with the synergist muscles, the upper trapezius, lower trapezius, and pectoralis major. The excessive activation of the upper trapezius muscle is one reason for abnormal scapular motion. ${ }^{2}$ It is possible to establish strategies for training the scapular stabilizing muscles, especially in patients with a low ratio of serratus anterior activation versus upper trapezius activation. Selective activation of the serratus anterior while minimizing activation of the upper trapezius might be advantageous, allowing for strengthening of the serratus anterior muscle in order to ease the imbalance in the serratus anterior activation versus upper trapezius activation ratio. ${ }^{8}$ The pectoralis major muscle can be activated simultaneously with the serratus anterior during active scapular exercises. ${ }^{14}$ It is also possible that the pectoralis major acts as a synergist muscle, compensating for a weak serratus anterior muscle. ${ }^{6,14}$

Piraua and colleagues concluded that performing push-up exercises on an unstable surface might be more favorable for producing higher levels of upper trapezius activity and lower levels of serratus anterior activity than doing push-up exercises on a stable surface. ${ }^{10}$ Many studies have confirmed that closed chain exercises, such as push-ups, can be used to strengthen the serratus anterior. However, the closed chain posture utilizes body weight, and this posture must be maintained with over-loading when the serratus anterior is activated as well as when other muscles, such as the upper trapezius and pectoralis major, are activated together. The open chain posture in the supine position can reduce the activation of other muscles because it shrinks the muscles by only supporting the weight of a subject's arm. Some studies found that the wall slide exercise is effective to activate selectively serratus anterior.$^{7,15}$ The wall slide exercise slip upward the lower arms against the wall with elbow flexed at $90^{\circ}$, the shoulder joints at $90^{\circ}$, and the trunk fixed in a standing posture. ${ }^{7}$ Although the wall slide exercise is not the same the posture of open chain exercise in this study, it does not have any weight bearing and focuses on the movement of a single joint, so it is similar the movement for the open chain exercise. Kim and Lim found that the wall side exercise might be effective in reducing pain and improving scapular alignment in subjects with scapular downward rotation rather than the sling slide exercise, such as closed chain posture. ${ }^{15}$ Hardwick and colleagues found that a wall slide exercise activated the muscle activity of serratus anterior than other traditional exercises. ${ }^{7}$

All the subjects in this study were healthy. If the subjects had weakness of the serratus anterior muscle, such as a winged scapula, the results could have been similar, or the differences between the two exercises might have been greater. The reason for this is that the serratus anterior is weak in a winged scapula, so activation of other muscles can occur more easily. Selective muscle activation requires delicate control and step-by-step exercise.

This study has a few limitations. The subjects were 13 healthy university students; thus, it is difficult to generalize its results. Moreover, the study used a cross-sectional research design, which did not include an intervention period for muscle strength training. Future research should study the long-term effects of open chain and closed chain exercises with winged scapula subjects.

\section{CONCLUSIONS}

This study found higher muscle activity in the serratus anterior and the lower pectoralis major, and a higher ratio of serratus anterior activation versus activation of the other 
two muscles in the open chain exercise posture. This study found that the open chain exercise in the supine posture selectively activates the serratus anterior better than the closed chain exercise in the quadruped posture. This finding suggests that the open chain exercise posture is a better than the closed chain exercise posture for selective activation of serratus anterior at the beginning of training.

\section{Key Points}

Question Is there the difference of serratus anterior muscle activity during protraction in open chain and close chain exercises in order to determine the posture that is best at selectively activating the serratus anterior muscle?

Findings The muscle activity of the serratus anterior was significantly higher and the activity of the pectoralis major was significantly lower in the open chain exercise than the closed chain exercise.

The ratio of serratus anterior versus other muscles were significantly higher in open chain exercise.

Meaning The open chain exercise in supine posture can activate selectively the serratus anterior rather than the closed chain exercise in quadruped posture.

\section{Article information}

Conflict of Interest Disclosures: None.

Funding/Support: None.

Acknowledgment: None.

\section{REFERENCES}

1. Lim JY, Kim TH, Lee JS. Reliability of measuring the passive range of shoulder horizontal adduction using a smartphone in the supine versus the side-lying position. $J$ Phys Ther Sci. 2015;27(10):3119-3122.

2. Sahrmann SA. Diagnosis and treatment of movement impairment syndromes. St Louis, MO: Mosby Inc; 2002.

3. Escamilla RF, Yamashiro K, Paulos L, et al. Shoulder muscle activity and function in common shoulder rehabilitation exercises. Sports Med. 2009;39(8):663-685.

4. Ludewig PM, Cook TM, Nawoczenski DA. Three- dimensional scapular orientation and muscle activity at selected positions of humeral elevation. J Orthop Sports Phys Ther. 1996;24(2):57-65.

5. Cools AM, Dewitte V, Lanszweert F, et al. Rehabilitation of scapular muscle balance: which exercises to prescribe? Am J Sports Med. 2007;35(10):1744-1751.

6. Decker MJ, Hintermeister RA, Faber KJ, et al. Serratus anterior muscle activity during selected rehabilitation exercises. Am J Sports Med. 1999;27(6):784-791.

7. Hardwick DH, Beebe JA, McDonnell MK, et al. A comparison of serratus anterior muscle activation during a wall slide exercise and other traditional exercises. $J$ Orthop Sports Phys Ther. 2006;36(12):903-910.

8. Ludewig PM, Hoff MS, Osowski EE, et al. Relative balance of serratus anterior and upper trapezius muscle activity during push-up exercises. Am J Sports Med. 2004; 32(2):484-493.

9. Martins J, Tucci HT, Andrade R, et al. Electromyographic amplitude ratio of serratus anterior and upper trapezius muscles during modified push-ups and bench press exercises. J Strength Cond Res. 2008;22(2):477-484.

10. Piraua AL, Pitangui AC, Silva JP, et al. Electromyographic analysis of the serratus anterior and trapezius muscles during push-ups on stable and unstable bases in subjects with scapular dyskinesis. J Electromyogr Kinesiol. 2014;24(5):675-681.

11. Kisner C, Colby LA. Therapeutic exercise: foundations and technique. 6th. Philadelphia: F.A Davis Com; 2012.

12. Cram JR, Kasman GS, Holtz J. Introduction to surface electromyography. Gaithersburg, Md.: Aspen Publishers; 1998.

13. Kendall FP, McCreary EK, Provance PG, et al. Muscles: Testing and function, with postural and pain. $5^{\text {th }}$. Baltimore: Lippincott Williams\&Wilkins; 2005.

14. Hiengkaew V, Wichaiwong K, Chaiyakul S, et al. Concerning the pectoralis major in active reaching exercise. Electromyogr Clin Neurophysiol. 2003;43(3):157-163.

15. Kim TH, Lim JY. The effects of wall slide and sling slide exercises on scapular alignment and pain in subjects with scapular downward rotation. J Phys Ther Sci. 2016;28(9):2666-2669. 